\title{
Encefalitis: ¿cuáles y cómo tratar?
}

\author{
ANTONIO BANFI P.*
}

\section{Encephalitis: Which are and how to treat?}

Viral infections, the main cause of encephalitis, invade CNS via bloodstream (e.: enterovirus), peripherical nerves (e.: rabies) and less frequently, via olfactory nerve (e.: free life amoebas). Focal neuron compromise explains symptomatic variability. In Chile no one virus predominates largely and epidemiological or clinical characteristics may confuse initially. Herpes simplex encephalitis is a neurological emergency which obligates an early diagnosis to initiate specific treatment. Herpes simplex type 1 is important in children and adults and type 2 in the neonate. Isolation of herpes simplex in CSF is scarce, PCR has about $91 \%$ sensibility and high specificity continuing positive until five or seven days after sympomatology and/or treatment have started. Electroencephalogram is sensitive but unspecific. Magnetic resonance image is the most useful image diagnosis in the early acute stage of disease; CT scan allows to control the evolution. Cerebral biopsy is reserved for patients without response to treatment. Acyclovir reduces mortality and sequels of herpes simplex encephalitis while higher acyclovir dose $(60 \mathrm{mg} / \mathrm{kg} /$ day $)$ has increased outcome in neonates. Valacyclovir and famcyclovir, with similar antiviral activity, could be employed to continue antiviral effect after 21 days of treatment. In enterovirus encephalitis dermatological manifestations or myocarditis may initially tend towards etiological diagnosis. Mycoplasma pneumoniae capacity to damage CNS is controversial, it can invade CSF but its pathological role subsists undefined. Less prevalent are HHV-6, Epstein-Barr virus, adenovirus, Bartonella henselae, Cryptococcus neoformans, Leptospira sp. and others. It is possible that new specific treatments may stimulate to develop technologies that allow us to explore better the etiology of acute encephalitis in our country.

Key words: Encephalitis; Differential diagnosis; Herpes simplex; Treatment

El escenario actual de las afecciones del sistema nervioso central (SNC) es complejo debido al creciente número de pacientes con diversas formas de inmunosupresión, el aumento de la sobrevida de los pacientes oncológicos, el fácil transporte de agentes patógenos de una a otra región mundial y el no menos importante problema de la resistencia microbiana.

Las encefalitis plantean siempre grandes dilemas los que abarcan todos los aspectos de la enfermedad: la presentación inicial, las manifestaciones clínicas, el diagnóstico, el tratamiento, la evolución y el pronóstico.

En este contexto el médico clínico se ve enfrentado a un paciente con un cortejo de síntomas de compromiso del SNC, habitualmente con una corta evolución, y entonces surge en su pensamiento una interrogante: ¿Estoy frente a un cuadro bacteriano o ante una encefalitis viral aguda?
Las encefalitis ocurren en todas las edades y pueden ser debidas a diversas causas infecciosas y de otra naturaleza; dependiendo de la causa el resultado final puede ser benigno o bien fatal. Las encefalitis de curso agudo son las más frecuentes y serán el objetivo principal del presente artículo. Las formas subagudas o crónicas de las encefalitis son propias de los pacientes inmunocomprometidos $y$, junto con las encéfalo-mielitis post infecciosas, constituyen entidades que no serán tratadas en esta ocasión.

Desde una perspectiva patológica, la encefalitis es un proceso inflamatorio que afecta al tejido cerebral y que casi siempre compromete las meninges; la causa más frecuente de encefalitis es la infección viral que determina inflamación perivascular y destrucción de la sustancia gris.

Debido al compromiso frecuente de las meninges se aceptan términos como meningo-

\footnotetext{
Departamento de Pediatría y Cirugía Infantil Oriente. Facultad de Medicina, Universidad de Chile. Servicio de Pediatría, Unidad de Infectología Hospital Luis Calvo Mackenna.

E-mail:abanfi@machi.med.uchile.cl
} 
Tabla 1. Principales etiologías virales de las encefalitis agudas

\begin{tabular}{ll}
\hline Virus & Virus \\
\hline Arbovirus & Herpes humano 8 \\
Citomegalovirus & Influenza \\
Coriomeningitis linfocitaria & Parotídeo \\
Enterovirus & Rabia \\
Epstein-Barr & Rubéola \\
Herpes simplex 1-2 & Sarampión \\
Herpes humano 6 & Varicela-zoster \\
& Virus de inmunodeficiencia humana \\
\hline
\end{tabular}

encefalitis o meningoencefalomielitis; sin embargo, ninguno de estos términos diferencia entre la inflamación aguda provocada por la acción directa de un agente causal o la derivada del proceso desmielinizante para o post infeccioso que puede comprometer las diferentes estructuras del SNC. Aquí reside gran parte de la incertidumbre que muchas veces está presente en los momentos de enfrentar inicialmente al enfermo y plantear un programa de manejo.

Los distintos agentes causales de encefalitis aguda (Tablas 1 y 2) provocan manifestaciones clínicas por compromiso directo o indirecto del sistema nervioso. La invasión del SNC puede ocurrir por vía sanguínea, la más frecuente, a partir de diversos sitios de entrada: respiratorio (virus varicela-zoster, virus parotídeo); intestinal (enterovirus), genital (virus herpes simplex o subcutáneo (arbovirus). Otra vía de invasión es a través de los nervios periféricos como en el caso de rabia. La penetración a través del nervio olfatorio que está representado en la mucosa nasal ha sido demostrada en las infecciones por amebas de vida libre que alcanzan el lóbulo frontal y el lóbulo olfatorio, luego de traspasar la lámina cribosa.

Uno de los hechos sustanciales en las encefalitis es el compromiso focal de las células nerviosas lo que explica las variaciones sintomáticas.

El estudio anátomo patológico de las encefalitis fatales ha permitido estudiar la reacción inflamatoria meníngea y perivascular cerebral, compuesta fundamentalmente de mononucleares. Entre otros cambios patológicos se aprecian procesos neuronales degenerativos, neuronofagia por los macrófagos y las células de microglia, y la presencia de células características conteniendo antígenos virales, como ocurre con la formación de células citomegálicas por el citomegalovirus.

La complejidad de las funciones cerebrales determina un variado conjunto de síntomas y signos que normalmente hacen muy difícil el diagnóstico diferencial.

En este proceso de diagnóstico, fundamental en el inicio de la enfermedad; la evolución temporal de la sintomatología y la localización anatómica juegan un papel primordial y el compromiso de otros órganos, si está presente, puede orientar a un diagnóstico preciso.

En el escenario inicial frente a un paciente surgen con fuerza algunas características generales de las encefalitis en nuestro medio y que hacen necesario tenerlas en cuenta: siendo los virus la principal etiología, ninguno de ellos predomina ostensiblemente como causa; la edad del paciente; la epidemiología ayuda sólo ocasionalmente; el cuadro clínico inicial puede corresponder a otras entidades no infecciosas; el examen citoquímico del LCR generalmente no orienta a la etiología y la existencia de diversos estudios de laboratorio que son específicos para cada agente

Tabla 2. Etiologías infecciosas no virales y no infecciosas, más frecuente en encefalitis aguda

\author{
Bacterias \\ Actinomyces sp. \\ Bartonella henselae \\ Brucella sp. \\ Chlamydia sp. \\ Legionella pneumophila \\ Listeria monocytogenes \\ Mycobacterium tuberculosis \\ Mycoplasma pneumoniae \\ Hongos \\ Cryptococcus $s p$. \\ Histoplasma $s p$. \\ Protozoos \\ Naegleria sp. \\ Plasmodium falciparum \\ Toxoplasma sp. \\ Misceláneas \\ Carcinoma \\ Vasculitis \\ Reacciones adversas a fármacos
}


obliga a un mayor esfuerzo por anticipar clínicamente la posible etiología; los recursos terapéuticos son escasos y surgen dilemas acerca de su uso adecuado. Una vez hecho este análisis inicial y establecido el manejo, se plantean dudas razonables acerca de la evolución y el pronóstico.

La importancia de ciertas características clínicas en encefalitis causadas por algunos agentes cobra interés en el momento de decidir el manejo del paciente. Pueden estar presentes signos y síntomas de inflamación meníngea pero, lo central para el diagnóstico clínico de encefalitis es el compromiso de conciencia, el que puede abarcar un amplio espectro. En ocasiones la alteración es leve pero los grados más profundos como la confusión, el estupor y el coma son característicos en la etapa aguda de los casos más definidos. Los signos de compromiso neurológico focal, las convulsiones, los reflejos osteotendinosos exaltados y la debilidad motora son también elementos claves.

La alteración inflamatoria del eje hipotálamo hipofisiario es responsable de la fiebre y el síndrome de secreción inapropiada de ADH. Si hay un componente inflamatorio medular es posible observar parálisis flácida y vejiga neurogénica además de hiporreflexia profunda. Otros signos son el edema papilar y el compromiso de nervios craneanos, especialmente el tercero y el sexto.

La encefalitis aguda de mayor importancia en clínica de adultos y niños es la herpética, no tanto por su incidencia como por la necesidad de establecer un diagnóstico precoz e instituir el tratamiento apropiado. Esta encefalitis es una emergencia neurológica y constituye la más seria de las manifestaciones de compromiso neurológico por VHS.

El VHS- 1 está asociado principalmente con la encefalitis de los niños y de los adultos mientras que el VHS-2 está relacionado con la encefalitis del recién nacido. En países con recursos diagnósticos disponibles generosamente, se reconoce la encefalitis herpética como la principal entidad no epidémica; en E.U.A. y otros países desarrollados, se estima una incidencia de 2 casos por millón de habitantes por año, sin diferencias estacionales en su presentación.

La importancia de su diagnóstico precoz reside en poder tratar oportunamente con aciclovir una entidad con una elevada letalidad y una serie de graves secuelas neurológicas en los sobrevivientes, si es que no se trata o es tratada tardíamente.

Los pacientes pueden tener un pródromo de malestar, fiebre, cefalea y náuseas. Posterior- mente presentan en forma aguda letargo, confusión y en ocasiones delirio. El cambio de personalidad es uno de los hechos llamativos en la encefalitis herpética denotando la predilección que tiene el virus por el lóbulo temporal. Las convulsiones, la afasia y otros signos focales pueden presentarse a continuación. En el examen neurológico pueden aparecer elementos de compromiso focal o global, parálisis de nervios craneanos y hemiparesia. Ocasionalmente se presentan signos meníngeos. Entre las presentaciones inusuales de la encefalitis herpética puede haber una forma subaguda, síndromes que imitan trastornos psiquiátricos y meningitis recurrente benigna. Menos frecuentemente, el VHS-1 produce una encefalitis del tronco cerebral y el VHS-2 una mielitis. Los pacientes inmunocomprometidos desarrollan una encefalitis necrosante más difusa, bi-hemisférica y con compromiso del tronco cerebral.

En los adultos la respuesta inmune combinada con factores virales condicionan la invasión y la virulencia, determinando una presentación y severidad muy variable. En los niños la encefalitis herpética es el resultado de una primoinfección en la mayoría de los casos.

En el recién nacido el riesgo de enfermedad por VHS es de $50 \%$ cuando la madre tiene una lesión primaria genital; de $33 \%$ cuando la lesión primaria es asintomática; de sólo $4 \%$ cuando la lesión es recurrente y el riesgo cae a $0,04 \%$ cuando existe el antecedente de una lesión herpética pero no hay evidencia de ella al examen ni la madre tiene manifestaciones prodrómicas.

El estudio citoquímico del LCR revela un aumento inespecífico de proteínas $(50-400 \mathrm{mg} / \mathrm{dL}$, $50 \%$ de los casos), una pleocitosis moderada (50-100 leucocitos $/ \mathrm{mm}^{3}$ ) de predominio mononuclear, una glucorraquia normal y la presencia de más de 10 hematíes $/ \mathrm{mm}^{3}$. La presencia de hematíes y la xantocromía representan una pista clínica en el momento inicial.

$\mathrm{El}$ aislamiento viral en el LCR tiene un bajo rendimiento en la encefalitis herpética y en la práctica el mayor avance diagnóstico, no sólo para VHS, es el estudio mediante la reacción de polimerasa en cadena (RPC). La RPC para VHS tiene una sensibilidad de no menos de $91 \%$ y una alta especificidad, permaneciendo positiva hasta cinco a siete días después de iniciada la sintomatología y/o el tratamiento. Los resultados falsamente positivos son escasos y se deben a contaminación cruzada en el laboratorio. Los resultados falsamente negativos, también escasos, pueden aparecer en recién nacidos y lactantes posiblemente debido a la presencia de inhibidores; 
además en la fase inicial de la encefalitis puede haber resultados falsamente negativos.

El electroencefalograma tiene $~ 84 \%$ de sensibilidad mostrando un patrón de trazado de espiga onda lenta con descargas epileptiformes lateralizadas y periódicas que surgen de las regiones temporales y fronto-temporales. Su especificidad es sólo de $32,5 \%$.

Entre los estudios imagenológicos la RM ha demostrado ser el mejor examen en las etapas iniciales al localizar lesiones en el lóbulo temporal y en el lóbulo oculotemporal. En cambio, en la TAC las lesiones pueden aparecer sólo al tercer o cuarto día de evolución y por lo tanto resulta más eficiente efectuar una TAC en el control de la evolución; en 50 a $75 \%$ de los pacientes muestra lesiones con áreas de baja densidad con efecto de masa en el lóbulo temporal, que posteriormente progresan a áreas radiolúcidas y hemorrágicas.

En la actualidad, con la disponibilidad de RPC inicial, la biopsia cerebral se reserva para aquellos casos en los cuales ha habido una falta de respuesta objetiva al tratamiento y surgen hallazgos distintos en la RM o TAC.

La biopsia muestra las inclusiones intranucleares de Cowdry en sólo $50 \%$ de los casos. Los efectos más definidos en la biopsia son la congestión capilar en la corteza cerebral y sustancia blanca, la necrosis y la inflamación.

El manejo médico de la encefalitis herpética contempla el soporte general, medidas de monitoreo de la hipertensión intracraneana (posición de la cabeza que favorezca el retorno venoso, manitol, apoyo intensivo) y el control de las convulsiones (fenitoina, carbamazepina). La participación del infectólogo en esta etapa es importante así como la del neurólogo. Si se está evaluando la posibilidad de biopsia cerebral, el neurocirujano resulta esencial.

El antiviral de elección es aciclovir que ha demostrado ser el más eficiente y menos tóxico en reducir, especialmente la letalidad y en menor grado, la morbilidad de la encefalitis herpética. Es un análogo sintético de purina. Inhibe la replicación viral selectivamente compitiendo con la guanosina por el ADN viral que codifica timidinoquinasa. In vitro su mayor actividad es frente a VHS-1, luego a VHS-2 y menor frente a varicela-zoster. Se han descrito mutantes resistentes de VHS en pacientes inmunosuprimidos de quienes se recuperan VHS deficientes en timidinoquinasa. Se excreta por vía renal y puede producir cristaluria en dosis altas que exceden su solubilidad. Para evitar este problema se debe cuidar la hidratación, la administración debe ser a velocidad moderada y se debe evitar el empleo conjunto de otros medicamentos nefrotóxicos. Otros efectos menos frecuentes son: flebitis, rash y alteraciones gastrointestinales. También tiene interacciones con fenitoina y carbamazepina disminuyendo sus concentraciones plasmáticas.

Una vez planteado el diagnóstico es mandatorio iniciar aciclovir intravenoso en dosis de 10 $\mathrm{mg} / \mathrm{kg} / \mathrm{dosis}\left(500 \mathrm{mg} / \mathrm{m}^{2} /\right.$ dosis $)$ en tres dosis diarias. La duración del tratamiento es de 14 a 21 días.

Recientemente se ha completado un estudio multicéntrico, empleando dosis mayores de 15 mg y $20 \mathrm{mg} / \mathrm{kg} /$ dosis durante 21 días en la infección diseminada por VHS en recién nacidos. Con la dosis mayor se obtuvieron resultados significativamente superiores, en términos de sobrevida con respecto al tratamiento clásico y los recién nacidos que sólo presentaron compromiso del SNC sobrevivieron en más de $90 \%$ de los casos.

El impacto sobre la morbilidad de este nuevo esquema no ha sido significativo y por otra parte, los niños tratados con dosis altas tienen una mayor incidencia de neutropenia por lo cual es necesario controlarlos periódicamente.

La letalidad anterior al empleo de aciclovir era de 60 a $70 \%$ y desde su introducción ha disminuido a $20 \%$ aproximadamente.

Posiblemente valaciclovir y famciclovir, ambos de administración oral y con actividad semejante a aciclovir puedan significar un avance al suceder en una fase oral de tratamiento al aciclovir intravenoso. En este sentido se están realizando estudios que pretenden analizar el efecto terapéutico de agregar valaciclovir con el fin de prolongar el tratamiento, más allá de los 21 días, mediante un medicamento oral con similar actividad que el aciclovir intravenoso.

Las complicaciones y secuelas ocurren en alrededor de $50 \%$ de los pacientes y las más frecuentes son el déficit motor, las convulsiones y los cambios mentales con deficiencias cognitivas y de memoria. Hay una estrecha relación entre el diagnóstico precoz, el inicio oportuno del tratamiento y el número de complicaciones y secuelas. Las alteraciones de memoria anterógrada y retrógrada y del lenguaje son secuelas que pueden presentarse aún con un tratamiento exitoso.

La encefalitis causada por enterovirus es generalmente un cuadro bien definido y cuando se presenta con signos precisos de focalización neurológica, no difiere mayormente en sus manifestaciones de la encefalitis herpética; sin embargo, otras manifestaciones como las cutáneas o la miocarditis pueden orientar el diagnóstico 
etiológico inicialmente. Entre los diversos enterovirus no poliovirus asociados con mayor frecuencia a compromiso del SNC están los echovirus 7, 9, 11 y 30; coxsackie B5 y enterovirus 71. Este último ha resultado especialmente preocupante al producir un cuadro de parálisis semejante a polio con letalidad de $14 \%$ y cuando se asocia compromiso miocárdico su letalidad es superior al $70 \%$.

El diagnóstico específico es complejo pues el cultivo de LCR es positivo en un bajo porcentaje debido a una escasa carga viral; además ciertos enterovirus requieren para su cultivo de animales de laboratorio. Así, la sensibilidad de los cultivos para enterovirus no sobrepasa 65 a $75 \%$.

La RPC es actualmente el procedimiento de laboratorio de elección en el diagnóstico de la encefalitis por enterovirus y tiene una especificidad de 94 a $100 \%$.

La encefalitis por enterovirus no tiene un tratamiento específico. El estudio del pleconaril, un inhibidor de cápside, en las infecciones por enterovirus produjo inicialmente entusiasmo al comunicarse buenos resultados especialmente en meningitis y ocasionalmente en encefalitis. Sin embargo, posteriormente sólo ha demostrado un efecto positivo en el manejo de las infecciones por rhinovirus.

Entre las etiologías que más controversia despiertan en el manejo de las encefalitis agudas está la causada por Mycoplasma pneumoniae. Aunque la asociación con enfermedad neurológica fue comunicada en 1943, aún se debate acerca de su capacidad de producir trastornos neurológicos, en particular los del SNC. No se discute la capacidad de acceder al LCR y se ha comprobado su persistencia en él por hasta ocho semanas. Lo que aún no se ha logrado definir es la respuesta inmune que provoca en el SNC. La meningoencefalitis estaría asociada a la invasión directa de la bacteria pero no se ha precisado el tiempo que media entre el inicio de los síntomas respiratorios, la entrada de $M$. pneumoniae al LCR y el comienzo de la encefalitis. Los síntomas son semejantes a otras encefalitis y generalmente son precedidos de una infección respiratoria; el diagnóstico lo sugieren las manifestaciones pulmonares y extrapulmonares como odinofagia y eritema multiforme, la presencia de deposiciones alteradas. La IgM específica es el diagnóstico rápido disponible; el cultivo es difícil y sólo el desarrollo y la difusión de la RPC en nuestro medio permitirá una precisión mayor.

Desde hace algunos años se ha definido el papel que desempeña el virus herpes humano (VHH-6) en el SNC; el agente causal de la roséola se ha asociado a convulsiones febriles y meningoencefalitis. Según distintos estudios, un amplio rango porcentual (0,5-50\%) de niños con exantema súbito presenta convulsiones febriles; también pueden desarrollar meningoencefalitis, hemiparesia, hemiplejía y secuelas en la esfera del desarrollo. El virus VHH-7 se ha asociado a hemiplejía aguda.

Una mayor evidencia del papel de VHH-6 en el SNC se ha obtenido con la determinación de la presencia de anticuerpos en el LCR y la detección de la secuencia génica del virus en el tejido cerebral de un niño con encefalitis y hepatitis fatal.

En algunos casos de niños que presentaron convulsiones febriles concomitantemente con la roséola, la reactivación del virus explicaría los nuevos episodios de convulsiones febriles que ocurren posteriormente.

La capacidad de provocar problemas neurológicos se amplía al considerarse que el VHH-6 tendría un posible papel en la esclerosis múltiple puesto que se ha demostrado que proteínas del virus se expresan en los oligodendrocitos de algunos pacientes.

El virus de Epstein-Barr también puede producir una meningoencefalitis, ataxia cerebelosa, mielitis transversa y neuropatía, que aparecen en el contexto de un cuadro típico de mononucleosis infecciosa o bien, con una menor definición clínica.

En la medida que en nuestro medio se desarrollen procedimientos de diagnóstico es evidente que se ampliará el espectro etiológico que actualmente contemplamos. Existe la necesidad de investigar el papel etiológico que tienen en nuestro medio agentes como adenovirus, Bartonella henselae, Cryptococcus neoformans, Leptospira $s p$ y otros.

Es posible que nuevas estrategias terapéuticas específicas impulsen el desarrollo de tecnologías que nos permitan conocer con mayor definición los reales agentes de encefalitis aguda en nuestro país.

\section{Resumen}

La causa más frecuente de encefalitis es la infección viral que invade el SNC por vía sanguínea ( $\mathrm{p}$ ej.: enterovirus), nervios periféricos ( $\mathrm{p}$ ej.: rabia) y menos frecuentemente, vía nervio olfatorio (p ej.: amebas de vida libre). El compromiso focal de las células nerviosas explica las variaciones sintomáticas. En nuestro medio ningún virus predomina ostensiblemente y las características epidemiológicas y clínicas pueden crear confusión inicial. La encefalitis herpética es una emergencia neurológica por la necesidad de un diagnóstico precoz, para instalar el tratamien- 
to específico. El VHS-1 es importante en niños y los adultos; VHS-2 en el neonato. El rendimiento del aislamiento viral en el LCR es bajo, la RPC tiene una sensibilidad aproximada de $91 \%$ y alta especificidad permaneciendo positiva hasta cinco a siete días después de iniciada la sintomatología y/o el tratamiento. El EEG es sensible pero poco especifico. La RM es el examen imagenológico indicado en etapa aguda; la TAC permite controlar la evolución. La biopsia cerebral se reserva para casos con falta de respuesta objetiva al tratamiento.

El aciclovir reduce la letalidad y las secuelas de la encefalitis herpética, dosis de $60 \mathrm{mg} / \mathrm{kg} / \mathrm{d}$ en recién nacidos han elevado su sobrevida. Valaciclovir y famciclovir, con similar actividad antiviral, podrían emplearse para prolongar el efecto antiviral más allá de 21 días.

En la encefalitis por enterovirus las manifestaciones cutáneas o la miocarditis pueden orientar el diagnóstico etiológico inicialmente.

Se debate la capacidad de Mycoplasma pneumoniae para producir trastornos del SNC, si bien invade el LCR, no se ha logrado definir su rol patógeno. Menos importantes son VHH-6, virus de Epstein-Barr, adenovirus, Bartonella henselae, Cryptococcus neoformans, Leptospira sp. y otros. Es posible que nuevos tratamientos específicos estimulen el desarrollo de tecnologías que nos permitan conocer mejor los reales agentes de encefalitis aguda en nuestro país.

\section{Bibliografía}

1.- Smalling T W, Sefers S E, Li H et al. Molecular approaches to detecting herpes simplex virus and enteroviruses in the central nervous system. J Clin Microbiol 2002 July; 40 (7): 2317-22.

2.- Kimberlin, D W, Lin, C Y, Jacobs, R F et al. Natural history of neonatal herpes simplex virus infections in the acyclovir era. Pediatrics 2001; 108: 223-9.

3.- Kimberlin D W, Lin, C Y, Jacobs R F et al. Safety and efficacy of high-dose intravenous acyclovir in the management of neonatal herpes simplex virus infections. Pediatrics 2001; 108: 230-8.

4.- Balfour H H. Antiviral drugs. N Engl J Med 1999; 340: 1255-68.

5.- Lahat E, Barr J, Barkai G et al. Long term neurological outcome of herpes encephalitis. Arch Dis. Child 1999; 80: 69-71.

6.- Aurelius E. Herpes simplex encephalitis: early diagnosis and immune activation in the acute stage and during long-term follow-up. Scand J Infect Dis 1993; 89: Suppl 3-626.

7.- Chaudhuri A, Kennedy P G E. Diagnosis and treatment of viral encephalitis. Postgrad Med J 2002; 78: 575-83.

8.- Ieven M, Demey H, Ursi D, et al. Fatal encephalitis caused by Mycoplasma pneumoniae diagnosed by the polymerase chain reaction. Clin Infect Dis 1998; 27: 1552-3.

9.- Atkins J T. HSV PCR for CNS infections: pearls and pitfalls. Pediatr Infect Dis J 1999 Sep; 18(9): 823-4.

10.- Domingues R B, Tsanaclis A M, Pannuti C S, et al. Evaluation of the range of clinical presentations of herpes simplex encephalitis by using polymerase chain reaction assay of cerebrospinal fluid samples. Clin Infect Dis $1997 \mathrm{Jul}$; 25(1): 86-91.

11.- Whitley R J, Lakeman F. Herpes simplex virus infections of the central nervous system: therapeutic and diagnostic considerations. Clin Infect Dis 1995 Feb; 20(2): 414-20.

12.- Whitley R J, Alford C A, Hirsch M S et al. Vidarabine versus acyclovir therapy in herpes simplex encephalitis. N Engl J Med 1986; 314(3): 144-9.

13.- Whitley R, Arvin A, Prober C et al. A controlled trial comparing vidarabine with acyclovir in neonatal herpes simplex virus infection. Infectious Diseases Collaborative Antiviral Study Group. N Engl J Med 1991; 324(7): 444-9.

14.- Whitley R J, Gnann J W. Viral encephalitis: familiar infections and emerging pathogens. Lancet 2002; 359(9305): 507-13.

15.- Caserta M T, Hall C B, Schnabel K et al. Neuroinvasion and persistence of human herpesvirus 6 in children. J Infect Dis 1994; 170(6): 1586-9.

16.- Yoshikawa T, Ihira M, Suzuki K et al. Invasion by human herpesvirus 6 and human herpesvirus 7 of the central nervous system in patients with neurological signs and symptoms. Arch Dis Child 2000; 83(2):170-1.

17. -Domachowske J B, Cunningham C K, Cummings D L et al. Acute manifestations and neurologic sequelae of Epstein-Barr virus encephalitis in children. Pediatr Infect Dis J 1996; 15(10): 871-5. 